\title{
Simulations of an innovative Time-of-Flight detector for high-energy neutrons based on iron-less RPCs
}

\author{
J. Machado ${ }^{a *}$, A. Blanco ${ }^{b}$, P. Fonte ${ }^{b, c}$, D. Galaviz ${ }^{a}$, L. Lopes ${ }^{b}$, R. Ferreira Marques ${ }^{b, d}$, \\ and P. Teubig ${ }^{a}$
}

${ }^{a}$ CFNUL, Centro de Física Nuclear da Universidade de Lisboa

1649-003 Lisboa, Portugal

${ }^{b}$ LIP, Laboratório de Instrumentacão e Física Experimental de Partículas

3004-516 Coimbra, Portugal

${ }^{c}$ ISEC, Instituto Superior de Engenharia de Coimbra

3031-199 Coimbra, Portugal

${ }^{d}$ Departamento de Física, Universidade de Coimbra

3004-516 Coimbra, Portugal

E-mail: jorgefdmecii.fc.ul.pt

In the upcoming years, radioactive beam facilities such RIBF (Tokyo, Japan), FRIB (East Lansing, USA) or FAIR (Darmstadt, Germany) will explore properties of unstable nuclei located within the limits of nuclear matter, the so-called "drip-lines". The detection of high energy neutrons is essential for the complete characterization of the reactions under study. In most cases a high-resolution neutron ToF spectrometer is required to determine the momentum of high-energy neutrons resulting from the decay of the projectile with energies in the range of $200 \mathrm{MeV}$ to $1000 \mathrm{MeV}$.

A novel concept for the detection of these relativistic neutrons is based on Resistive Plate Chambers (RPCs). The detection principle of the RPC-based detector relies on the detection of the charged particles created in hadronic showers induced by the incoming neutrons. The presented design only considers glass plates for holding the active gas acting simultaneously as converters for neutron detection. For the optimization of a large area detector based on RPCs several simulations were performed using the Virtual Monte Carlo framework FAIRROOT. The detector was designed as a structure of single RPCs modules with 5 gas gaps grouped sequentially reaching a total efficiency for one neutron detection higher than $90 \%$. In this work we present the results of the simulations carried out and the evaluation of the performance of the detector concept.

XI workshop on Resistive Plate Chambers and Related Detectors - RCP2012,

February 5-10, 2012

INFN Laboratori Nazionali di Frascati Italy

\footnotetext{
* Speaker.

${ }^{\dagger}$ Work supported by Portuguese FCT, project PTDC/FIS/114876/2009
} 


\section{Introduction}

The upcoming generation of nuclear physics facilities will study the properties of neutronrich nuclei close to the limits of the existence of nuclear matter. The investigations of reactions with unstable nuclei in inverse kinematics will require the use of a high-resolution neutron ToF spectrometer to determine the momentum of high-energy neutrons emitted by the projectile, with energies in the range of 200 to $1000 \mathrm{MeV}$.

In this work we present a new approach for the detection of single neutrons (i.e. no more than one neutron is emitted in the reaction process) at relativistic energies. In order to impose a control on the performance of the device, a momentum resolution of $\Delta p / p \leq 10^{-3}$ was requested. Such a performance can be achieved considering a time resolution of $\sigma_{t}<100 \mathrm{ps}$ and a position resolution of $\sigma_{x, y, z} \approx 1 \mathrm{~cm}$ for flight paths in the range from 10 to $35 \mathrm{~m}$. In addition, the system is designed to reach a minimum detection efficiency for one neutron of $90 \%$. The performance of the detector was simulated using the R3BROOT package [1] within the FAIRROOT framework [2, 3], developed at GSI.

\section{The RPC-based detector}

Based on the requirements for the detector, the utilization of RPCs for the design is very attractive. This type of detector is based on common materials and large tRPC (timing RPC) can provide good time and position resolutions [4]. Besides, an efficiency of more than $95 \%$ for minimum ionizing particles (MIPs) can be achieved.

Since neutrons have no charge, the detection principle follows the creation of charged secondary particles by nuclear reactions induced in the converter material. First simulations concentrated on the performance of the RPC module in the detection of neutrons interacting with different converter materials: paraffin $(15 \% \mathrm{H}, 85 \% \mathrm{C})$, for which the main reaction principle are inelastic collisions with hydrogen; and steel $(71 \% \mathrm{Fe}, 18 \% \mathrm{Cr}, 11 \% \mathrm{Ni})$, in which secondary particles are expected to result from direct reactions with iron. In addition, the thickness of the converter plates and the total depth of the detector, expressed in number of planes, were also varied. The design of the RPC considered during these simulations is shown in Fig. 1 for the case of using steel as the converter material. In this design, the central part contains twice the thickness of the considered nominal converter thickness.

The dependence of the efficiency on the thickness of the converter material for steel is shown in Fig. 2. As a probe, neutrons of $800 \mathrm{MeV}$ were sent to the detector. All configurations, for a certain converter thickness and a specific number of planes, reach a maximum efficiency, which then decreases with increasing converter thickness. For small converter thicknesses, the efficiency increases with the number of planes. Depending on the detector configuration, there is a certain thickness value, for which the efficiency starts to decrease, as the particles created in the steel do not have enough energy to escape and reach the RPC gas. A similar study performed with paraffin as converter material gave significantly different results as it can be seen in Fig. 3. The increase in efficiency with increasing converter thickness is less peaked than in the steel case. However, it never decreases for the considered range of thicknesses. 

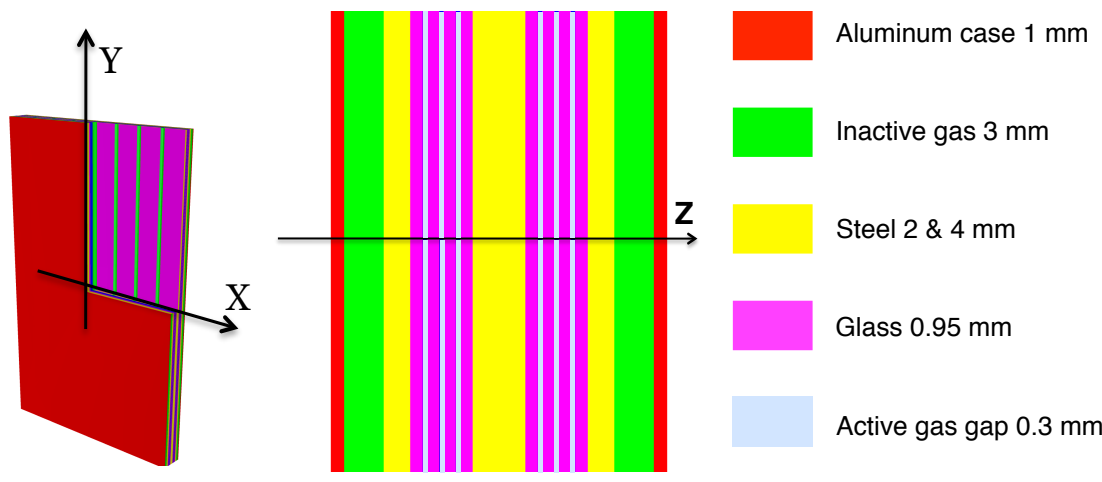

Figure 1: (Color online) Schematic view of the RPC design considered in the study of the performance of different converter material and converter thickness. The presented design considers steel plates of $2 \mathrm{~mm}$ thickness as converter [5].

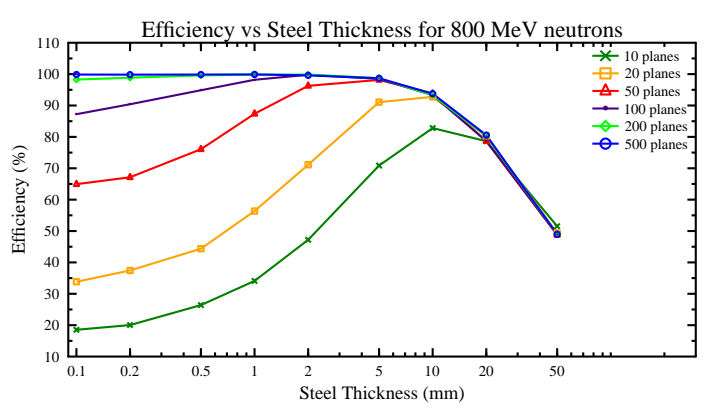

Figure 2: (Color online) Dependence of the detection efficiency on the thickness of the steel converter for several configurations with different number of detector planes.

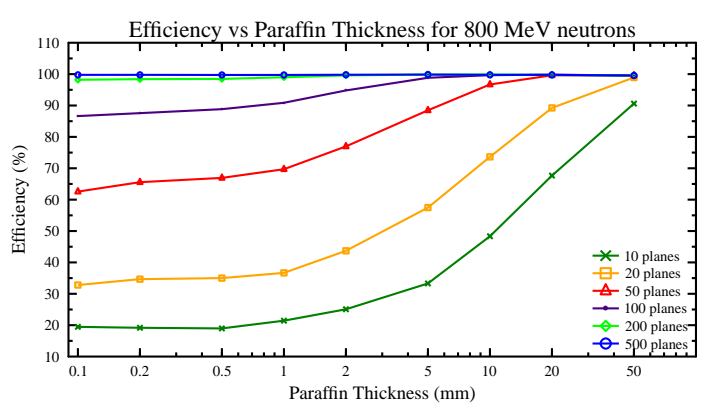

Figure 3: (Color online) Dependence of the detection efficiency on the thickness of the paraffin converter for several configurations with different number of detector planes

In both cases, when considering negligible thicknesses of the converter material (i.e., no converter), the detection efficiency is non-zero and has equivalent values leading to the idea, that the rest of the components of the detector, predominantly the glass, also work as converters. This provided the input for the new approach of using an iron-less concept in the design of the RPC.

\section{The Iron-less concept}

Based on the fact that the glass plates holding the active gas work as a converter material, a new concept for the detection of high-energy neutrons was developed: the iron-less RPC. Fig. 4 shows a schematic diagram of the adopted structure of the RPC.

The RPC module is consists of 5 gas gaps and 6 glass plates, all contained inside a gas tight case made of plastic with feedthroughs for gas flow and HV. Based on this geometry for the RPC, two parameters need to be optimized: the thickness of the individual glass plates, and the total number of planes required to meet the initial efficiency criterium.

Configurations considering glass thicknesses between 1 and $5 \mathrm{~mm}$ were studied. The dependence of the efficiency on the number of planes for each configuration is shown In Fig. 5. 

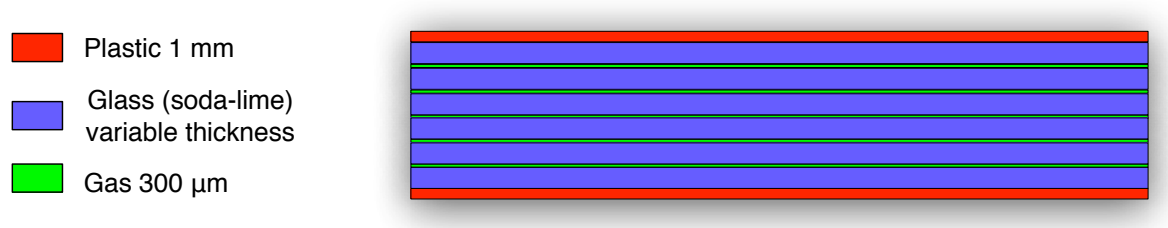

Figure 4: (Color online) Schematic view of the iron-less RPCs. Six glass plates hold the active gas encapsulated in a tight plastic box.

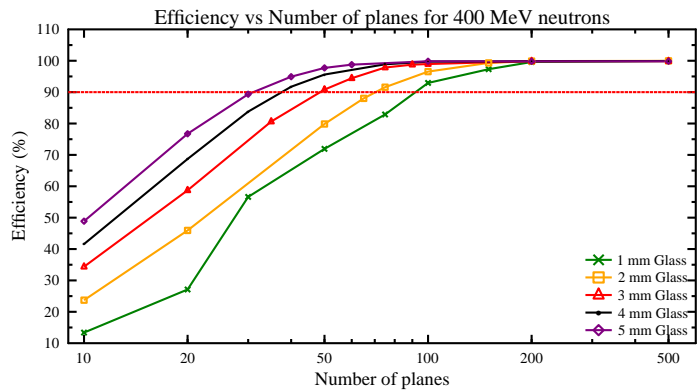

Figure 5: (Color online) Dependence of the detection efficiency on the number of planes considered for the different configurations under study (glass plates with thickness between 1 and $5 \mathrm{~mm}$ ). After a certain number of planes, all configurations reach and go beyond $90 \%$ efficiency.

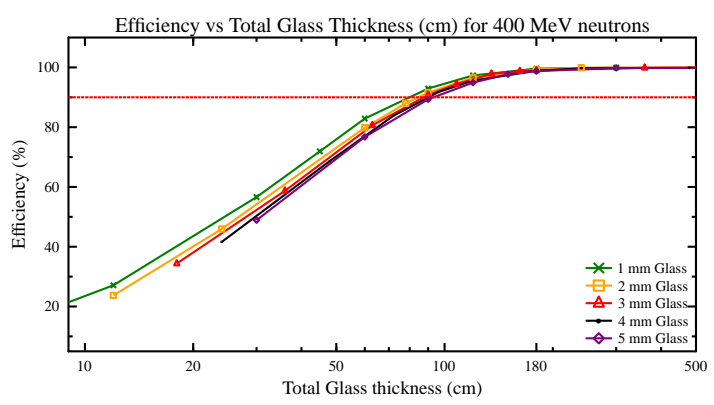

Figure 6: (Color online) Dependence of the detection efficiency on the total amount of glass contained in the RPC detector. This parameter seems to be the key factor for the neutron detection efficiency. Maximum efficiency can be reach with a total glass thickness of $180 \mathrm{~cm}$.

As can be seen, for all those glass plates thicknesses the design goal of $90 \%$ efficiency for one neutron detection is reached. However, considering this particular RPC design, the detection efficiency can be parameterized as a function of the total glass thickness in the whole detector shown in Fig. 6. The overlaying curves point to the fact that a direct relationship between efficiency and total glass thickness exists, which was not the case for the two other converter materials. Besides, all configurations reach a $100 \%$ efficiency for a total glass thickness of $180 \mathrm{~cm}$ independently of the thickness of the individual plates.

Furthermore, simulations were performed to investigate the performance of the different configurations considering various glass plate thicknesses and number of planes with the preferred configuration being the $3 \mathrm{~mm}$ glass plates and 100 planes [6].

The response of this particular configuration to monoenergetic neutrons was simulated. Neutrons with $400 \mathrm{MeV}$ were impinging on the detector at normal incidence angle. The result of the simulation was folded with random functions to reproduce the spacial and time uncertainties expected by such a module. As for the $\mathrm{x}$ and $\mathrm{z}$ coordinates, a square random distribution of $3 \mathrm{~cm}$ (size of the readout strip) and $1.075 \mathrm{~cm}$ for the $\mathrm{z}$ (half size of the plane thickness) was applied. For the $\mathrm{y}$ and for the time, gaussian distributions of $\sigma_{y}=1 \mathrm{~cm}$ and $\Delta_{t}=80 \mathrm{ps}$ were applied, respectively.

The deviation of the reconstructed momentum with respect to the original one, $\Delta p=p_{\text {derived }}-$ $p_{\text {input }}$, is shown in Fig. 7. As it can be seen, the reconstruction of the momentum of the neutrons presents a $\sigma$ resolution of about $1.5 \mathrm{MeV} / \mathrm{c}\left(\Delta p / p \approx 1.510^{-3}\right)$, which is in agreement with the 
design goals for the detector.

$\Delta p$ for $3 \mathrm{~mm}$ glass 100 planes at $35 \mathrm{~m}$

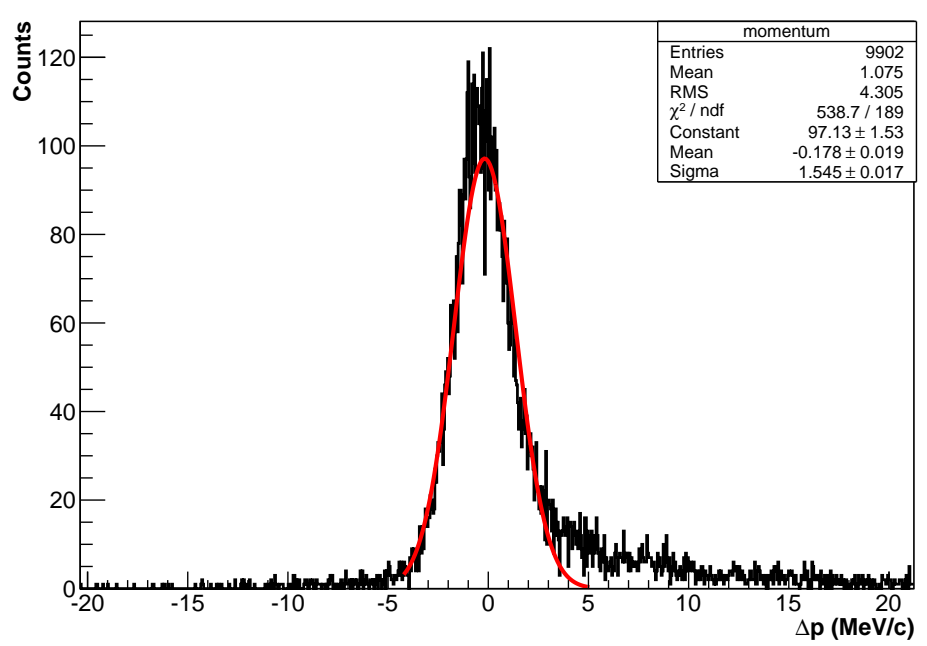

Figure 7: Deviation of the reconstructed momentum with respect to the original one, $\Delta p=p_{\text {derived }}-p_{\text {input }}$, in the measurement with $400 \mathrm{MeV}$ neutrons. The detector is located at $35 \mathrm{~m}$. The $\sigma$ of the distribution shown is approximately $1.5 \mathrm{MeV} / \mathrm{c}$, in agreement with the design goals of the detector.

\section{Prototype Simulations}

The breakup of relativistic deuterons on a proton target will allow the characterization of several detector prototypes using "monoenergetic" neutrons at GSI. In the forthcoming experiment S406 [7], neutrons with an energy ranging between 200 and $1500 \mathrm{MeV}$ are expected to be measured by various neutron detection prototypes and by the LAND detector.

Concerning the design presented in this work, a prototype with a total active area of $200 \times 50$ $\mathrm{cm}^{2}$ consisting of 4 modules of 5 gas gaps and 4 modules of 2 gas gaps, will be characterized. The thickness of the glass plates will be $3 \mathrm{~mm}$. This configuration should provide very relevant information on how the hadronic showers are generated inside an RPC module and its transmission along the detector. In addition, valuable data on the time response of the RPC to the interaction with neutrons should be obtained.

The design of the considered prototype has been simulated, placing the detector at the closest possible distance to the target $(5 \mathrm{~m}$ ) and considering $400 \mathrm{MeV}$ neutrons. The expected uncertainty in the momentum distribution is shown in Fig. 8. The expected momentum resolution turns out to be $8.5 \mathrm{MeV} / \mathrm{c}$, about a factor 5 larger that expected for the whole detector.

\section{Conclusions}

Simulations on the performance of an RPC as a neutron detector have been performed, investigating the dependence on the type of neutron converter material and its thickness. Based on this study, a new concept for the RPC based exclusively on glass plates has been developed and studied. Overall, simulations show an equivalent performance when compared to the RPC with steel. 


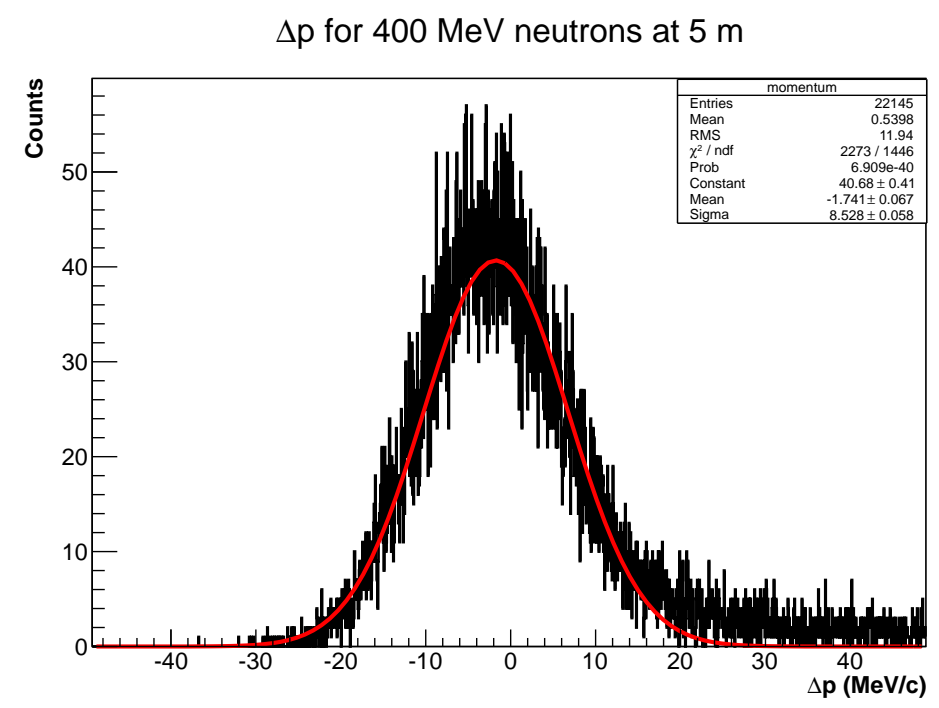

Figure 8: Deviation of the reconstructed momentum with respect to the original one, $\Delta p=p_{\text {derived }}-p_{\text {input }}$, in the measurement with $400 \mathrm{MeV}$ neutrons by the prototype detector to be tested at GSI. The prototype is located at $5 \mathrm{~m}$. The $\sigma$ of the distribution shown is about $8.5 \mathrm{MeV} / \mathrm{c}$

Simulations using the iron-less prototype to be tested during experiment S406 at the GSI laboratory were also performed. The prototype is currently under construction. The analysis of the data will be compared to the simulation results, so a deeper understanding on the interaction of relativistic neutrons with the RPC can be achieved.

\section{References}

[1] D. Bertini, "R3broot: a ROOT based simulation and analysis framework for $\mathrm{R}^{3} \mathrm{~B}$," GSI Annual Report, 2009.

[2] D. Bertini, M. Al-Turany, I. Koenig, and U. Florian, "The FAIR simulation and analysis framework," Journal of Physics: Conference Series 119 032011, 2008.

[3] Al-Turany, D. Bertini, R. Karabowicz, and F. Uhlig, "Status of the FAIRROOT simulation and analysis framework," GSI Annual Report, 2010.

[4] A. Blanco, R. Ferreira-Marques, C. Finck, P. Fonte, A. Gobbi, A. Policarpo, and M. Rozas, "A large area timing RPC," Nuclear Instruments and Methods in Physics Research Section A: Accelerators, Spectrometers, Detectors and Associated Equipment, vol. 485, p. 328, 2002.

[5] Z. Elekes, "private communication."

[6] J. Machado, "Simulations of an innovative Time-of-Flight detector for high-energy neutrons," $U L$, Master Thesis, 2011.

[7] K. Boretzky, "Characterization of NeuLAND prototypes and the land detector using fast "monoenergetic" neutrons," proposal for SIS experiment S406, 2010. 